\title{
Introduction to Blockchains and Cryptocurrencies
}

\author{
Sam Goundar \\ The University of the South Pacific, Suva, Fiji \\ sam.goundar@gmail.com
}

\begin{abstract}
Blockchains and cryptocurrencies are now topics of substantial impact that academia, practitioners and the IT industry need to contemplate, study, research, publish, innovate, exploit and adopt. This book titled Blockchain Technologies, Applications and Cryptocurrencies intends to provide information on the innovative, scholarly and professional research pertaining to the management, organization and technological use of blockchains and cryptocurrencies. The chapters in this book will be essential for anyone interested in these areas. This book is one of the few dedicated entirely to blockchain technologies, applications and cryptocurrencies.
\end{abstract}

Blockchain technologies are being claimed to be as disruptive as the Internet. A white paper, written by Satoshi Nakamoto (a pseudonym), "Bitcoin: A Peer-to-Peer Electronic Cash System" appeared in 2008, setting everything into motion. The white paper detailed how an electronic cash transaction can take place between peers without the need for a third party (financial institution). Since then, the paper has been cited 5,687 times at the time of writing this introduction. Digital signatures and cryptography would be used to secure the financial transaction, and peers on 
the bitcoin network running on the blockchain technology would verify, validate and authorise the transaction to eliminate the "double spend problem".

According to Nakamoto (2008), this would make the system and transaction transparent, immutable and under the control of peers, thus eliminating control of financial institutions and their exorbitant fees. The bitcoin network would timestamp transactions by hashing them into an ongoing chain of hash-based proof-of-work, forming a record that cannot be changed without redoing the proof-of-work. The longest chain will not only serve as proof of the sequence of events witnessed, but proof that it came from the largest pool of CPU power. Messages are broadcast on a best effort basis, and nodes can leave and rejoin the network at will, accepting the longest proof-of-work chain as proof of what happened while they were gone.

The website BlockGeeks.Com (2019) defines "blockchain" as a growing list of records, called blocks, which are linked using cryptography. Each block contains a cryptographic hash of the previous block, a timestamp and transaction data (generally represented as a Merkle tree). By design, a blockchain is resistant to modification of the data. It is "an open, distributed ledger that can record transactions between two parties efficiently and in a verifiable and permanent way". Blockchain use with peer-to-peer electronic cash transactions enabled a number of Financial Technology (FinTech) applications, Distributed Ledger Technology (DLT) applications and introduction of more than thousand other Cryptocurrencies. Bitcoin still remains the most popular cryptocurrency.

Blockchains have now moved from electronic cash to other applications in government, supply chain management, healthcare, agriculture, real estate, international development and almost any application that utilize databases that can be replaced with a more secure, immutable, consensus-based, transparent and trust-based database. Apart from FinTech applications, other applications based on blockchains are emerging in every sector and industry as everyone is intent on taking advantage of the special properties of blockchains mentioned in the previous sentence. After bitcoin's success or failure (it depends), people are trying to apply it to procedures and processes beyond financial transactions. In effect, they are asking: What other agreements can a blockchain automate? What other middlemen can blockchain technology retire? 
Some examples of real-world blockchain applications in use are as follows:

- Land Ownership and Management: Dubai, USA, UK, Sweden, Ukraine, Georgia and Ghana.

- Development Aid: Aid Transfer Efficiency, South African Early Childhood Development.

- Supply Chain Management: Diamond Tracking (De Beers), Food Safety (Walmart), Oil Supply (ADNOC), Agricultural Products Supply (LDC).

- Renewable Energy: WePower (Estonia), Power Ledger (Australia), Acciona Energy (Spain), The Brooklyn Micro Grid (US), The Sun Exchange (South Africa).

- Remittances: Blockchain Wallet, TransferWise, Ripple's XRP, BitSpark, MoneyFi, Chynge.

- Digital Identity: Civic, ValidatedID, THEKEY, Trusti, PeerMountain, Edge, BlockAuth, BlockVerify, CryptID, ExistenceID.

- Agriculture: Food Traceability, Fair Trade Farming, Organic Farming Certifications, Food Safety (Pesticides Use), New Markets, Logistics.

- Democracy and Governance: Voting, Decentralisation of Services, GovCoin, Democracy.Earth, FollowMyVote, Smart Participation, Liquid Democracy.

- Manufacturing and Industrial: Provenance, JioCoin, Hijro, SKUChain, BlockVerify, STORJ.io, RFID Integration, Anti Counterfeiting, Compliance.

- Health: Modum.io, Gem, SimplyVital Health, MedRec, Electronic Health Records (EHR), AmbrOSys, Hashed Health, Medical Change, Change Health.

- Financial Inclusion: MojaLoop, ABRA, Bank Apoalim, Maersk, Augur, Regalii, Ripple, World Remit, Stellar, Oridian, Credits.Vision, OneName, ShoCard.

- Retail: OpenBazaar, Loyyal, Blockpoint.io, Customer Data Management, Transparency, Warranty, Goods Tracking, Customer Loyalty.

- Climate Change: Power Ledger, ClimateCoin.io, Carbon Emission Trading, Clean Energy, GHG. 
- Environment: SOLshare, PlasticBank, Agora Tech Lab, EnergiToken, CarbonX, Veridium, IBM, United Nations, Waste Reduction, GiveTrack.

- Human Rights: Stop the Traffik, Dash Venezuela, Micro Trade, OpenGarden, RightMesh, Media Freedom, Social Media Awareness.

- Access to Water: Clean Water Coin, Decentralized Water Management, Water Quality Assurance Regulations, Water Trading.

- Cyber-security: GuardTime, REMME, IoT Security, Decentralized Storage Solutions, Safer DNS, Security in P2P Messaging systems.

Cryptocurrencies are "digital money" that do not physically exist but can be converted to any popular physical currency. Bitcoin, the first digital money, was hatched as an act of defiance. Unleashed in the wake of the Great Recession, the cryptocurrency was touted by its early champions as an antidote to the inequities and corruption of the traditional financial system. Bitcoin sought to replace the services provided by financial institutions with cryptography and code. When you pay your mortgage, a series of agreements occur in the background between your financial institution and others, enabling money to go from your account to someone else's. Bitcoin and other cryptocurrencies replace those background agreements and transactions with software — specifically, a distributed and secure database called a Blockchain.

If you could piece together a running tabulation of who held every dollar, then suddenly the physical representations would become unnecessary. Bitcoin achieved the running tabulation by creating a single, universally accessible digital ledger called a blockchain. Bitcoin's blockchain, unlike the ledgers maintained by traditional financial institutions, is replicated on networked computers around the globe and is accessible to anyone with a computer and an Internet connection. A class of participants on this network, called miners, is responsible for detecting transaction requests from users, aggregating them, validating them and adding them to the blockchain as new blocks. It's called a chain because changes can be made only by adding new information to the end. Each new addition, or block, contains a set of new transactions - a couple of thousand that reference previous transactions in the chain.

"CIO Insights Reflections: Cryptocurrencies and Blockchains their Importance in the Future" by Notling \& Muller (2017) reports the 
following: "With the help of cryptography and a collective booking system called Blockchain, cryptocurrencies build a distributed, safe and decentralized payment system, which does not need banks, intermediates, an organization or a central technical infrastructure to work. The main difference to the current types of money we know is that an intermediate, which is responsible for production (e.g. central bank) or exchange (banks) is not needed. Exchanges of digital values and goods are made directly between two individuals." Known cryptocurrencies are Bitcoin, Ethereum, Ripple, Litecoin and IOTA. In a sense, they are scarce commodities as the amount of available currency units is in this case limited by mathematical algorithms. After every digital currency unit is issued, there is no way to generate additional currency units from it (e.g. Bitcoin is limited to 21 million units). Furthermore, every cryptocurrency has its own currency generating process. The main factors likely to affect the future development of cryptocurrencies are, in Notling \& Muller's (2017) opinions, interventions by the government and central banks and questions on how the sector will be regulated.

The chapters in this book are as follows:

\section{Chapter 1: A Literature Review in Support of Blockchain Technologies}

Blockchain technology has to be one of the biggest innovations of the 21st century given the ripple effect it is having on various sectors from financial to manufacturing as well as education. Unknown to many is the fact that Blockchain history dates back to the early 1990s. Since its popularity started growing a few years back, a number of applications have cropped up all but underlining the kind of impact it is destined to have as the race for digital economies heats up. This chapter examines the literature on Blockchain technologies and potential benefits of Blockchain technologies wherever it is used. This research is being carried out because there is no literature review on Blockchain Technology, and so, this chapter will ease the research of the scholars who want to know about Blockchain Technology. This research is important because this chapter will make people aware about Blockchain and all the places it is used and how people can benefit from this technology. Search terms identified more than 30 papers in support of blockchain technologies. The methodology used to carry out the research is also discussed in this chapter, such as 
various databases that were searched using different search terms. From the papers identified, specific criteria were then applied to identify appropriate articles. Data analysis verified the purpose of blockchain, categories of blockchain and alternatives to blockchain. The findings revealed that blockchain is one of the leading technologies in the world currently that possesses one of the more secure platforms for carrying out any task.

\section{Chapter 2: A Taxonomy of Blockchain Applications}

A taxonomy of blockchain application discusses cases - uses and apps. A major obstacle identified in several disciplines is the categorization of objects in a particular area of interest into a specific taxonomy. Blockchain is an innovation of decentralized and value-based information distributed over a vast network that contains untrustworthy users. Developing a taxonomy for blockchain application is a complex process, and thus, the purpose of this chapter is to discuss what internal factors contribute to the success of a blockchain application. This is followed by a discussion of which organizations use blockchain and which applications and apps they use. Finally, the chapter aims to classify blockchains according to what they are used for, e.g. blockchains are used in finance, agriculture, education, and research and development.

\section{Chapter 3: Blockchain Means More Than a Software to Democracy: Access to Fundamental Rights of Sixth Dimension}

Throughout history, five dimensions of basic needs have been created to establish the existential minimum. This chapter aims to demonstrate that Human Rights attained the position of the Sixth Dimension in 2009 when the right of access to technology became fundamental to generating peace, dignity and a sustainable world. Establishing technology as a fundamental right is useful to guide the Annual Budget Plan developed by the leaders who use these fundamental rights to establish the margin of investment to be made in each sector proportional to the level of importance it occupies in a citizen's life. For these reasons, research has been done which brought to light the fact that that blockchain and cryptocurrencies are much more than payment systems. The method used was scientific with analysis being done of historical documents as well as social observation and seeking of laws, doctrines and jurisprudence confronted with data taken from 
socioeconomic statistical reports collected in 2017. This research was enough to show that the Blockchain Technology and Cryptocurrencies combined with AI and IoT is the Smart Economy. Through poverty reduction, physical borders will be protected by virtual borders. The authors conclude that technologies are a powerful accelerator of economic flow and act as a vehicle for the sustainable world requiring investments like Human Rights being the Sixth Dimension, one that cannot be separated from others' rights.

\section{Chapter 4: Autonomous Agriculture Marketing Information System Through Blockchain: A Case Study of e-NAM Adoption in India}

The National Agricultural Market, through networking of the APMCs, provides electronic trading for agricultural produce. Under e-NAM, all the trading is to be done through a digital platform as prescribed to ensure transparency in the transactions and provide a fair price to the farmers. The study was conducted in three APMCs of Uttar Pradesh to analyse the ground-level practices taking place in these APMCs and level of adoption of e-NAM. It was found that there is a considerable variation in the arrival and bidding prices obtained from the APMCs when compared with the data available on the agriculture market information system (Agmarknet). The study proposes a blockchain-based infrastructure to facilitate a more transparent, autonomous system to empower the information system and efficient application of government rules and regulations pertaining to agricultural transactions in the APMCs through the utilization of smart contracts.

\section{Chapter 5: Attack Vectors for Blockchain and Mapping OWASP Vulnerabilities to Smart Contracts}

Smart contracts powered by Blockchain render transaction processes more effective, secure and efficient when compared with conventional contacts. Smart Contracts facilitate trustless process, time efficiency, cost effectiveness and transparency without any intervention by third-party intermediaries like lawyers. While Blockchains can counter traditional cyber-security attacks on Smart Contract Applications in a significantly good manner, cyber-criminals keep creating new threats and attack vectors that can hack Blockchain technologies. This chapter presents a unique framework to perform Application Security Testing on Blockchain-based 
Smart Contracts and compares Manual Penetration Testing with two automated Smart Contract tools to detect critical vulnerabilities on a commercial production Blockchain environment.

\section{Chapter 6: Blockchain Application in Fiji's Aviation Industry}

In today's high-technological era, organizations have accelerated the use of blockchain technology to enhance their business processes. Blockchain is somewhat an updated version of database, which is a powerful technology in terms of managing data when compared with normal SQL databases. In Fiji, flight information is a mission critical system as a large number of stakeholders depend on it, such as hotels, airlines, airports, ground handlers and, overall, the general public. Current flight information is meeting the day-to-day organizational needs, but there are information technology problems associated with it, such as inconsistent information, data silos, and availability. With the introduction of blockchain technology, the aviation industry can become more powerful in terms of providing high-quality flight data to all its stakeholders. By the end of the research, we should be able to build a conceptual model that solves the flight data problem in Fiji.

\section{Chapter 7: Blockchain-Based e-Voting Application}

Voting has been the fundamental part of a democratic system as it allows individuals to voice out their opinions. For the past few years, voting turnout has diminished as concerns regarding security, privacy, accessibility and integrity escalated. In order to address these issues, e-voting was introduced; however, only few countries managed to use the application due to cost and central authority approvals. Hence, blockchain technology is an emerging platform as it allows decentralization through the use of distributed technology, thereby expanding industries and processes. In this chapter, the researchers discuss the significance of blockchain e-voting application as well as provide details of the issues faced by current blockchain technologies. The chapter will also provide a comparative analysis of existing mechanisms in order to understand and mitigate the gaps before fully adopting blockchain technologies for e-voting applications. Thus, this chapter will be a roadmap for blockchain-based e-voting application to improve the current voting practices and processes. 


\section{Chapter 8: Blockchains for Supply Chain Management Networks}

Blockchain is a distributed and digital ledger which has transformed supply chain in various ways. In this chapter, we will investigate how blockchain can add value to the supply chain management (SCM) system. This chapter will further investigate the influence of integrating blockchain technology to the current supply chain system and its long-term implications. In essence, the research has been carried out to verify if blockchain technology is capable of providing the transparency and the accountability the current SCM systems lacks. The chapter looks at advantages and disadvantages of such integration and provides feedback and recommendation on the same. The research findings aim to provide a better insight into current practices used by large logistic and supply industries and what the future holds for such companies using supply chain management system.

\section{Chapter 9: Comparison of Three Different Darknet Cryptocurrencies in e-Commerce in Our Digital Era}

The emergence of the internet has opened a world of possibilities to connect and interact through web portals to exchange information over the network. In the past decade, buying and selling of goods and services online has become more common through e-commerce. With the advent of Cryptocurrencies, the possibilities of e-commerce have reached new heights for all web users who see potential in this technology. Cryptocurrencies have opened new dimensions based on a Blockchain technology for e-commerce through decentralization — increased privacy and digitalization of coins to allow users to anonymously trade online. This has become more common in the Darknet, which is simply the unindexed side of the Internet. This paper compares the three Cryptocurrencies, namely Bitcoin, Ethereum and Monero, in the Darknet used for e-commerce.

\section{Chapter 10: Cryptocurrencies - An Assessment of Global Adoption Trends}

Government and state approaches to any venture first begin with regulation to safeguard its interests as well as those of its citizens. Cryptocurrency adoption globally has been led by major players such as 
the U.S and China while some smaller states have achieved comparable success. In this chapter, we analyse and assess a country's level of cryptocurrency adoption via its regulatory framework. We then classify this approach according to the business function that regulates the use of cryptocurrencies. We also derive a gradual approach to cryptocurrency adoption and regulation that can be used by fledgling countries new to the world of cryptocurrency.

\section{Chapter 11: An Overview of Cryptocurrencies for Online Payments of Enterprise Systems}

Cryptocurrencies have evolved erratically and at unprecedented speed over the course of their short lifespan. Since the inception of Bitcoin in 2009 , more than 1,600 cryptocurrencies have been developed with majority successful in the market. With the advent of cryptocurrencies, the possibility of online trading has reached new heights. Thus, this chapter focuses on enterprise cryptocurrencies and provides a thorough analysis of the various opportunities that lie in the usage of cryptocurrencies for online payments. This chapter was prepared as there is a need to draw a fine line between using fiat currencies and using cryptocurrencies for online transactions, as there is not much research done on using cryptocurrencies in Enterprise systems, and this chapter will give a fair idea to researchers and organizations in making decisions while making corporate payments. We are basing our research using grounded theory, and focus on the certainty of using cryptocurrencies.

As the editor, writer and the co-author of all chapters of this book, I am proud to present this book to the readers. I would like to thank all the reviewers that peer-reviewed all the chapters in this book. I also would like to thank the administration and editorial support staff of World Scientific Publishing who have ably supported me in getting this book to press and publication. And finally, I would like to humbly thank all the authors and co-authors who submitted their research articles as chapters to this book. Without your submission, your tireless efforts and contribution, we would not have this book.

Publication of any book is not easy. Publication of a new book on a relatively new technology with a reputable publisher is even harder. The first step is to find a publisher that is willing to publish such a book. Given 
the dynamics of academic publishing today, disruptive transformation of academic publishing by the Internet, delivery and access of published research papers by PDF downloads, and Open Access academic journal models, many established publishers are now reluctant to publish a new book. This book on Blockchain Technologies, Applications and Cryptocurrencies is indeed privileged to be published by World Scientific Publishing - a publisher that is second to none. Disruptive technologies like blockchains and cryptocurrencies have been seen by many as a passing fad. The publication of this book is proof that they are not a fad but a mainstream disruptive technology.

I hope everyone will appreciate reading the chapters in this book. Additionally, I feel that it will inspire and encourage readers to start their own research on blockchains and cryptocurrencies and submit their articles and chapters to journals and books.

Once again, I take this opportunity to congratulate everyone involved in the writing, review, editing and publication of this book.

Any comments or questions can be emailed tosam.goundar@gmail. com.

Happy Reading.

\section{References}

Blockgeeks (2019). What is Blockchain Technology? A Step-by-Step Guide for Beginners. Retrieved from: https://blockgeeks.com/guides/what-is-blockchain-technology/. Accessed on May 19, 2019.

Nakamoto, S. (2008). Bitcoin: A Peer-to-Peer Electronic Cash System.

Notling, C., Muller, M. (2017). CIO Insights Reflections: Cryptocurrencies and Blockchains - Their Importance in the Future. Deutsche Bank Wealth Management. Retrieved from: https://www.db.com/newsroom_news/cio_ insights_reflections_-_cryptocurrencies_and_blockchains_-_EMEA_-_ client_ready.pdf. Accessed February 29, 2020. 